\title{
VARIAÇÃO DE ATRIBUTOS FÍSICO-HÍDRICOS EM LATOSSOLO VERMELHO-AMARELO DO CERRADO MATO-GROSSENSE SOB DIFERENTES FORMAS DE USO(1)
}

\author{
Geovani José Silva(2), Daniel Dias Valadão Júnior ${ }^{(3)}$, Aloísio \\ Bianchini $^{(4)}$, Emílio Carlos de Azevedo ${ }^{(4)}$ \& João Carlos de \\ Souza Maia ${ }^{(4)}$
}

\begin{abstract}
RESUMO
A busca de uma produção que atenda às crescentes demandas mundiais, em consonância com a manutenção das potencialidades dos recursos naturais, numa visão sistêmica de sustentabilidade social, econômica e ambiental, deve ser a meta de todos e, em especial, dos profissionais das ciências agrárias. Com o objetivo de contribuir nesse sentido, avaliou-se o efeito da produção no sistema plantio direto e da pastagem cultivada sobre os atributos físico-hídricos de um Latossolo Vermelho-Amarelo distrófico. $\mathrm{O}$ estudo foi realizado em uma propriedade agrícola localizada sob as coordenadas de $15^{\circ} 31^{\prime} 58$ " $\mathrm{S}, 55^{\circ} 18,07$ ' W, altitude entre 725 e $809 \mathrm{~m}$, relevo suavemente ondulado, em áreas com os seguintes usos: plantio direto (PD) por oito anos, pastagem de Brachiaria humidicola (PC) por sete anos consecutivos e Cerrado nativo pastejado (CP). Dez pontos, escolhidos aleatoriamente em cada área, foram submetidos a ensaio de resistência mecânica do solo à penetração (RSP) e amostrados nas profundidades de 05-10 e 10-15 cm. Nas amostras deformadas, determinou-se o conteúdo de água atual (CA), a textura e a densidade de partícula (Dp), e, a partir das amostras indeformadas, foram determinadas a porosidade total (Pt), macroporosidade (Ma), microporosidade (Mi), condutividade hidráulica saturada (Ks), as curvas características de retenção de água e a densidade do solo (Ds), bem como o conteúdo de água disponível (AD).
\end{abstract}

\footnotetext{
(1) Escrito a partir de relatório de pesquisa orientada da disciplina de Física do solo do Programa de Pós-graduação em Agricultura Tropical - FAMEV/UFMT. Recebido para publicação em janeiro de 2008 e aprovado em junho de 2008.

${ }^{(2)}$ Doutorando do Programa de Pós-graduação em Agricultura Tropical, Faculdade de Agronomia e Medicina Veterinária, Universidade Federal do Mato Grosso - FAMEV/UFMT. Av dos Ramires s/n, CEP 78200-000 Cárceres (MT). Bolsista CAPESPICDTEC. E-mail: gjsilva@ufmt.br

(3) Mestrando do Programa de Pós-graduação em Agricultura Tropical, FAMEV/UFMT. Bolsista CNPq. E-mail: jrdias@ufmt.br

(4) Professor Adjunto do Departamento de Solos e Engenharia Rural, FAMEV/UFMT. Av. Fernando Corrêa da Costa s/n, Coxipó, CEP 78060-900 Cuiabá (MT). E-mails: bianchi@ufmt.br; emilioaz@ufmt.br; jotace@terra.com.br
} 
Os dados foram submetidos a análises de variância e a teste de comparação de médias $(p<0,05)$. O delineamento experimental foi inteiramente casualizado, em esquema de parcelas subdivididas ( 3 usos $x 2$ profundidades). $O$ solo sob PD sofreu acréscimo de até $13,38 \%$ na Ds, 45,83 \% na RSP, 29,81 \% na AD e decréscimo de até 45,61\% na Ma e de até 91,89\% na Ks. Os efeitos sobre os atributos do solo ocorreram na seguinte ordem de magnitude: $\mathrm{PD}>\mathrm{PC}>\mathrm{CP}$. A RSP, Ma e Ks foram os atributos mais afetados pelos efeitos do uso do solo.

Termos de indexação: plantio direto, pastagem, resistência do solo à penetração.

\title{
SUMMARY: CHANGES IN HYDRO- PHYSICAL ATTRIBUTES OF A TYPIC HAPLUSTOX IN THE CERRADO OF MATO GROSSO STATE UNDER DIFFERENT FORMS OF SOIL USE
}

\begin{abstract}
The search for production forms that meet the increasing worldwide demands, maintaining the potential of the natural resources and in a systemic view of the social, economic and environmental sustainability, must be everybody's interest and above all a concern of professionals of agrarian sciences. Aiming at a contribution in this direction, the effect of no-till and cultivated pasture production on the physical and water properties of a Typic Haplustox (Distrophic Red-Yellow Latosol) were evaluated. The study was conducted on a private farm (lat $15^{\circ} 31^{\prime} 58^{\prime} \mathrm{S}$, long, 55 ${ }^{\circ} 18^{\prime} \mathrm{O} 77^{\prime} \mathrm{W}$, at $726-809 \mathrm{~m}$ asl) in a gentle sloping topography. Ten randomly chosen points in a maize field under no-till management ( $P D$ for eight years), Brachiaria humidicola pasture (PC - grazed for seven consecutive years), and native Cerrado pasture (CP) were tested for soil resistance to mechanical penetration (RSP) and sampled in the layers 5-10 and 10-15 cm. In the disturbed samples the current water content $(C A)$, particle texture and density $(D p)$ were determined, and in the undisturbed samples the total porosity (Pt), macroporosity (Ma) and microporosity (Mi), saturated soil water conductivity $(K s)$, characteristic curves of soil water retention, and bulk density $(D s)$, as well as the available water content $(A D)$. The data were submitted to analysis of variance and the averages compared at $p<0.05$, using a completely randomized design with split plots (3 soil uses $\mathrm{x} 2$ sampling depths). In the soils under PD an increase of up to $13.38 \%$ was observed in Ds, $45.83 \%$ in SRP, $29.81 \%$ in AD and decreases of up to $45.61 \%$ in Ma and up to $91.89 \%$ in Ks. The magnitude of effects on soil properties were ranked as follows: $P D>P C>C P$. The $R S P, M a$ and Ks were the attributes most affected by the distinct soil uses.
\end{abstract}

Index terms: no-tillage, pasture, soil penetration resistance.

\section{INTRODUÇÃO}

A grande inquietação da agricultura atual está na busca da sustentabilidade da exploração agrícola tanto no âmbito social quanto ambiental, ou de uma nova condição de equilíbrio do sistema de produção, o que envolve vários fatores, entre eles o manejo adequado do solo (Soares et al., 2005).

No estado natural, os solos possuem características químicas e físicas definidas, em razão da rocha matriz, dos processos pedogenéticos, da vegetação e da topografia. Inevitavelmente, o desflorestamento e a mecanização continuada podem desequilibrar o sistema solo e o meio e modificar suas propriedades químicas, físicas e biológicas, limitando sua utilização agrícola e tornando-o mais suscetível à erosão (Centurion et al., 2001).

Com o intuito de diminuir a mecanização, minimizar a erosão e possibilitar o plantio em solos arenosos, passou-se a utilizar em larga escala a semeadura feita com o mínimo preparo do solo, ou o plantio direto. Neste sistema, a ausência quase completa de preparo resulta em maior qualidade do solo em relação ao preparo convencional (D'Andréa et al., 2002); contudo, os atributos físicos do solo sofrem alterações, independentemente do uso.

Em solos manejados sob o sistema plantio direto, Da Ros et al. (1997) observaram aumento da densidade, da porosidade total e do tamanho de agregados, e Camargo \& Alleoni (1997) relataram aumento da 
resistência à penetração já nos primeiros anos de cultivo. O aumento da densidade ocorre, em média, dos 7 aos $15 \mathrm{~cm}$ (Vieira, 1981), enquanto o aumento da resistência geralmente ocorre entre as profundidades de 10 e $30 \mathrm{~cm}$, como relatam FreitasJúnior \& Luchiari-Júnior (1994). Essas modificações devem-se principalmente à utilização de equipamentos agrícolas e à repetitividade das operações ao longo dos anos (Oliveira, 2002). Ainda segundo esse autor, o tráfego de máquinas e a ausência de revolvimento promovem alterações na estrutura do solo, principalmente na macroporosidade na profundidade entre 0 e $5 \mathrm{~cm}$.

O uso do solo para formação de pastagens tem sido outra forma de exploração bastante difundida e que ocupa grande extensão de terras. Nessas áreas destinadas ao pastejo, a principal causa de alterações nas propriedades físicas do solo relaciona-se ao pisoteio animal. A magnitude do efeito do pisoteio animal na compactação do solo depende, principalmente, da categoria e da carga animal (Moraes \& Lustosa, 1997). Em solo de Cerrado sob pastoreio, notam-se alterações nas propriedades físicas, como aumento na densidade, diminuição no tamanho dos agregados estáveis em água e redução da macroporosidade, bem como redução no conteúdo de matéria orgânica (Melo \& Silva, 1995). As alterações ocasionadas pela pressão do pisoteio animal ocorrem especialmente na camada entre $3 \mathrm{e}$ $6 \mathrm{~cm}$ de profundidade, segundo Gradwell (1966).

A dinâmica da água no solo também é uma característica modificada de acordo com o uso do solo, por ser dependente de fatores como a porosidade (quantidade, continuidade e tamanho), uma vez que a compactação e a descontinuidade dos poros reduzem a permeabilidade, implicando empobrecimento e perda de solo pela erosão hídrica (Souza \& Alves, 2003).

Assim, o objetivo deste trabalho foi avaliar as alterações dos atributos físico-hídricos de um Latossolo Vermelho-Amarelo distrófico sob Cerrado, quando submetido ao cultivo de soja e milho no sistema plantio direto e ao uso com pastagem de Brachiaria humidicola.

\section{MATERIAL E MÉTODOS}

A fase do experimento no campo foi realizada em abril de 2007, em uma área com Latossolo VermelhoAmarelo distrófico textura franco-arenosa, profundo, relevo plano, pertencente à "Agropecuária Rio Manso". Esta fazenda, com área de 3.006 ha, localiza-se no município de Campo Verde-MT, sob coordenadas de $15^{\circ} 31^{\prime} 58^{\prime \prime} \mathrm{S}$ e $55^{\circ} 18^{\prime} 07$ " W, e possui altitude entre 725 e $809 \mathrm{~m}$ e relevo variando entre plano e suave ondulado.

O clima é tropical continental semi-úmido, tipo Aw, conforme Köppen (1948), com período seco de maio a setembro e grande parte da precipitação anual média, de $1.421 \mathrm{~mm}$, concentrada em dezembro, janeiro e fevereiro. A temperatura média anual é de $25,6^{\circ} \mathrm{C}$, ocorrendo em outubro a média mensal mais elevada $\left(27,2^{\circ} \mathrm{C}\right)$ e, em julho, a menor $\left(22,8^{\circ} \mathrm{C}\right)$.

Adotou-se delineamento experimental inteiramente casualizado, com seis tratamentos e dez repetições, em esquema de parcela subdividida, em que as parcelas foram os usos do solo (vegetação nativa Cerrado, plantio direto e pastagem cultivada) e as subparcelas, as profundidades de 5 a 10 e 10 a $15 \mathrm{~cm}$.

$\mathrm{Na}$ área de plantio direto (PD), continuamente há oito anos, a soja é semeada sobre restos de milheto, e o milho "safrinha" é cultivado sobre os resíduos da soja. A área de pastagem cultivada (PC) é formada com Brachiaria humidicola e, há sete anos, o solo foi escarificado, gradeado e corrigido com calcário e fosfato. Portanto, em razão do longo período sem manutenção, apresenta sinais de degradação, presença de plantas autóctones e baixa produção de forragem. A lotação nessa área girou em torno de 1,0 UA ha-1 em aproximadamente dez meses do ano. Como referência, adotou-se uma área de Cerrado nativo (CP) contígua à área de pastagem, que, embora não receba nenhuma outra intervenção, é disponibilizada ao gado para pastoreio durante oito meses no ano, sob uma lotação estimada em cerca de 0,3 $\mathrm{UA} \mathrm{ha}^{-1}$.

As profundidades de amostragem foram definidas a partir dos resultados de ensaios preliminares de penetrometria, realizados aleatoriamente em 10 pontos de cada área. Com base na curva de resistência mecânica do solo à penetração média, de cada tipo de uso do solo, foram selecionadas as profundidades, de forma que se obtivesse melhor estratificação vertical do solo em estudo.

Em cada uma das áreas selecionadas, foram escolhidos, aleatoriamente, 10 sítios de amostragem, que foram identificados e tiveram a resistência mecânica do solo à penetração (RSP) medida por meio de um penetrógrafo eletrônico automático manual com velocidade de penetração constante, desenvolvido por Bianchini et al. (2002). Foram realizados quatro ensaios em pontos afastados cerca de $0,50 \mathrm{~m}$ do centro de cada sítio, sendo a resistência registrada a cada $2,5 \mathrm{~mm}$, até a profundidade de $300 \mathrm{~mm}$.

No centro de cada sítio de amostragem, nas camadas de 5 a 10 e 10 a $15 \mathrm{~cm}$ de profundidade, coletaram-se amostras com estrutura preservada, utilizando amostrador de Kopeck, com anel metálico de $50 \mathrm{~mm}$ de diâmetro e $50 \mathrm{~mm}$ de altura, num total de 60 amostras. Além das amostras indeformadas, coletaram-se também amostras deformadas, utilizando-se trado holandês, na camada de $0-20 \mathrm{~cm}$, para determinar a textura e, nas mesmas profundidades das amostras indeformadas, para determinação do conteúdo de água do solo no momento da coleta. As amostras coletadas foram acondicionadas de forma que mantivessem suas características originais e transportadas para o Laboratório de Física de Solo da Faculdade de Agronomia e Medicina Veterinária, da Universidade Federal de Mato Grosso, 
na cidade de Cuiabá, onde foram analisadas conforme os métodos recomendados pela Embrapa (1997).

Os atributos analisados foram: densidade do solo (DS), resistência mecânica do solo à penetração (RSP), porosidade total (Pt), macroporosidade (Ma), microporosidade (Mi), condutividade hidráulica saturada $(\mathrm{Ks})$ e conteúdo de água disponível (AD); foi também determinada a curva de retenção de água no solo. Para caracterizar as parcelas e minimizar possíveis erros, determinou-se também a textura, a densidade de partícula (Dp) e o conteúdo de água do solo (CA) no momento da amostragem. A análise textural foi feita pelo método do densímetro de Boyoucos, sendo o dispersante $\mathrm{NaOH} 0,1 \mathrm{~N}$. Determinou-se a densidade de partículas $(\mathrm{Dp})$ pelo método do balão volumétrico, utilizando álcool etílico como líquido penetrante, para obtenção do volume do solo.

A DS foi determinada pelo método do anel volumétrico. A Pt foi a soma da macro e da microporosidade do solo, que foram obtidas pelo método da mesa de tensão a $6 \mathrm{kPa}$. A Ks foi determinada pelo método da coluna constante de água, e o CA, pelo método-padrão (gravimétrico). Considerou-se como água disponível aquela retida no solo entre as tensões de 6 e 1.000 kPa (Silva \& Resck, 1981).

Para obtenção da curva de retenção, duas amostras indeformadas, por tratamento, foram previamente saturadas e submetidas às tensões de 50, 100, 300, $500,1.000$ e $1.500 \mathrm{kPa}$, em câmaras extratoras de Richards. Os dados relativos ao conteúdo de água nas diferentes tensões foram processados utilizandose o software Soil Water Retention Curve - SWRC (Dourado Neto et al., 2000), para obtenção das curvas de retenção ajustadas, conforme a equação: $\theta=\theta r+$ $\left\{\theta s-\theta r /\left[1+(\alpha \psi)^{n}\right]^{m}\right\}$, em que $\theta$ : umidade volumétrica do solo $\left(\mathrm{cm}^{3} \mathrm{~cm}^{-3}\right)$; $\theta r$ e $\theta s$, umidade residual e de saturação do solo, respectivamente $\left(\mathrm{cm}^{3} \mathrm{~cm}^{-3}\right) ; \psi$ : potencial matricial da água no solo $\left(\mathrm{cm} \mathrm{H}_{2} \mathrm{O}\right)$; e $\alpha, n$, $m$, parâmetros de ajuste do modelo de van Genuchten (1980).

Os dados foram submetidos à análise de variância, e as médias dos tratamentos, comparadas pelo teste de Skott-Knott, adotando-se como critério de significância de $\mathrm{F}$ e de diferença entre médias valores de $\mathrm{p}<0,05$. Os dados foram processados utilizandose o software SISVAR 4.6 (Ferreira, 2000).

\section{RESULTADOS E DISCUSSÃO}

Os resultados da análise textural dos solos na camada de 0-20 cm, nas áreas com os três diferentes usos, permitem admitir semelhança na textura deles, uma vez que apresentaram teores médios das frações minerais da ordem de 196,3 $\pm 4,3 \mathrm{~g} \mathrm{~kg}^{-1}$ de argila, $13,9 \pm 0,0$ de silte e 789,8 $\pm 13,7$ de areia total.

No quadro 1, pode-se observar a significância de F na análise de variância dos atributos físico-hídricos do solo, de acordo com os três usos e as duas profundidades estudadas. Os coeficientes de variação (CVs) foram relativamente baixos para esse tipo de experimento, em que geralmente são observados CVs bem maiores - como exemplo, têm-se os encontrados por Nielsen et al. (1973), com valores entre 6, 46 -10 \% para a Ds, e por Bertol et al. (2001), com valores de 16,4 a $74 \%$ para a RSP. Os maiores coeficientes de variação foram observados para a variável condutividade hidráulica saturada $(\mathrm{Ks})$, estando de acordo com Souza \& Alves (2003). Segundo Lima \& Silans (1999), a Ks é um dos atributos com maior coeficiente de variação, alcançando valores de CV da ordem de 226,10 \%, como encontrado por Azevedo (2004) em solo sob pastagem. Afirmam os autores que essa variação seria dependente da macroporosidade, da

Quadro 1. Resultado da análise de variância dos atributos físico-hídricos de um Latossolo Vermelho-Amarelo distrófico, em função do uso do solo e da profundidade de amostragem

\begin{tabular}{|c|c|c|c|c|c|}
\hline \multirow{2}{*}{ Atributo $^{(1)}$} & \multicolumn{3}{|c|}{ Fonte de variação } & \multirow{2}{*}{ CV (US) } & \multirow{2}{*}{$\mathrm{CV}(\mathrm{P})$} \\
\hline & Uso do Solo (US) & Profundidade (P) & US $\times \mathbf{P}$ & & \\
\hline & & Significância de F & & - & - \\
\hline Ds $\left(\mathrm{Mg} \mathrm{m}^{-3}\right)$ & 0,0000 & 0,0104 & 0,0370 & 2,09 & 2,09 \\
\hline $\mathrm{RSP} \quad(\mathrm{MPa})$ & 0,0000 & 0,0001 & 0,0000 & 8,06 & 4,95 \\
\hline $\mathrm{CA} \quad\left(\mathrm{cm}^{3} \mathrm{~cm}^{-3}\right)$ & 0,0000 & 0,2884 & 0,8281 & 8,62 & 5,54 \\
\hline $\mathrm{AD}\left(\mathrm{cm}^{3} \mathrm{~cm}^{-3}\right)$ & 0,0001 & 0,0005 & 0,0086 & 15,24 & 8,27 \\
\hline $\mathrm{Ks} \quad\left(\mathrm{mm} \mathrm{h}^{-1}\right)$ & 0,0000 & 0,0000 & 0,0000 & 26,08 & 18,88 \\
\hline $\mathrm{Pt} \quad\left(\mathrm{cm}^{3} \mathrm{~cm}^{-3}\right)$ & 0,0018 & 0,0726 & 0,1087 & 8,46 & 3,75 \\
\hline $\operatorname{Ma}\left(\mathrm{cm}^{3} \mathrm{~cm}^{-3}\right)$ & 0,0000 & 0,0040 & 0,0024 & 12,37 & 8,75 \\
\hline $\operatorname{Mi}\left(\mathrm{cm}^{3} \mathrm{~cm}^{-3}\right)$ & 0,0000 & 0,0002 & 0,0078 & 7,57 & 3,91 \\
\hline
\end{tabular}

DS: densidade do solo; RSP: resistência mecânica do solo à penetração; CA: conteúdo atual de água do solo; AD: água disponível; Ks: condutividade hidráulica saturada; Pt: porosidade total; Ma: macroporosidade; Mi: microporosidade. 
distribuição de partículas e da Ds, além da existência de infiltrações preferenciais em macroporos ou ao longo das raízes. Souza \& Alves (2003) atribuíram os elevados coeficientes de variação desse atributo ao efeito local, em conseqüência da alta variabilidade espacial dos solos, típica das propriedades de movimentação tridimensional da água.

Ainda no quadro 1, verifica-se o efeito significativo dos tratamentos sobre todos os atributos avaliados, a 0,01 e a 0,05 de probabilidade, mostrando assim que o uso do solo e a profundidade propiciaram alterações dos atributos físico-hídricos. Somente a profundidade e a interação entre uso e profundidade não apresentaram significância $(p<0,05)$ em relação aos atributos CA e Pt, mostrando, nesse caso, que essas fontes de variações não influenciaram as alterações desses atributos.

Observa-se que o CA apresentou níveis de significância relativamente altos (valores de $\mathrm{p}$ bem superiores a 0,05 ) para profundidade e interação usoprofundidade, mostrando que a umidade no momento da coleta não apresentou variações significativas em profundidade, condição essencial para obtenção da RSP, uma vez que esta é fortemente influenciada pelo conteúdo de água no solo.

No caso da $\mathrm{Pt}$, a ocorrência de valores de $\mathrm{F}$ nãosignificativos para o tratamento profundidade e a interação uso-profundidade demonstra não ter havido alterações no total de poros do solo entre as camadas amostradas. Entretanto, a profundidade de coleta acarretou alterações na distribuição do tamanho de poros, visto que os valores de $\mathrm{F}$ foram altamente significativos $(\mathrm{p}<0,01)$ para os atributos Ma e Mi.

Ao se fazer uma análise geral dos valores médios dos atributos físico-hídricos do solo (Quadro 2), verificase que não houve diferenças significativas com relação à profundidade de coleta nas áreas com plantio direto e pastagem cultivada. As exceções, nesses casos, referem-se a RSP em ambos os usos, $\mathrm{AD}$ em plantio direto e Ks em pastagem cultivada. Já nos solos sob $\mathrm{CP}$, ao contrário, ocorreram variações em quase todos os atributos avaliados, excetuando-se o conteúdo atual de água (CA). Isso demonstra o efeito da ação antrópica, do manejo e do uso dos solos, no sentido de homogeneizar as camadas de 5-10 e de $10-15 \mathrm{~cm}$ de profundidade, tornando-as similares com relação aos valores dos atributos em estudo.

As áreas sob PD e PC apresentaram valores estatisticamente superiores de Ds, RSP e Mi e inferiores de $\mathrm{Ks}$, Pt e Ma, em relação ao CP, mesmo apesar de esta última ter sido visitada regularmente pelo gado, evidenciando a compactação dos solos nas áreas manejadas (PD e PC).

Os valores médios de densidade do solo (Quadro 2) variaram de $1,42 \mathrm{Mg} \mathrm{m}^{-3}$ na camada de $10-15 \mathrm{~cm}$ do solo sob Cerrado a $1,61 \mathrm{Mg} \mathrm{m}^{-3}$ em ambas as camadas

Quadro 2. Médias dos valores dos atributos físico-hídricos de um Latossolo Vermelho-Amarelo distrófico, em função da profundidade de amostragem e do uso (Plantio Direto com soja/milho - PD), pastagem cultivada (PC) e Cerrado nativo pastejado (CP)

\begin{tabular}{|c|c|c|c|c|}
\hline \multirow{2}{*}{ Atributo } & \multirow{2}{*}{ Profundidade } & \multicolumn{3}{|c|}{ Uso do solo } \\
\hline & & PD & PC & $\mathbf{C P}$ \\
\hline & $\mathrm{cm}$ & & & \\
\hline $\mathrm{Ds}\left(\mathrm{Mg} \mathrm{m}^{-3}\right)$ & $\begin{array}{l}05-10 \\
10-15\end{array}$ & $\begin{array}{l}1,61 \mathrm{Aa} \\
1,61 \mathrm{Aa}\end{array}$ & $\begin{array}{l}1,52 \mathrm{Ba} \\
1,50 \mathrm{Ba}\end{array}$ & $\begin{array}{l}1,47 \mathrm{Ca} \\
1,42 \mathrm{Cb}\end{array}$ \\
\hline RSP (MPa) & $\begin{array}{l}05-10 \\
10-15\end{array}$ & $\begin{array}{l}2,07 \mathrm{Bb} \\
2,45 \mathrm{Aa}\end{array}$ & $\begin{array}{l}2,29 \mathrm{Aa} \\
1,80 \mathrm{Bb}\end{array}$ & $\begin{array}{l}1,93 \mathrm{Ca} \\
1,68 \mathrm{Cb}\end{array}$ \\
\hline $\mathrm{CA}\left(\mathrm{cm}^{3} \mathrm{~cm}^{-3}\right)$ & $\begin{array}{l}05-10 \\
10-15\end{array}$ & $\begin{array}{l}0,138 \mathrm{Aa} \\
0,138 \mathrm{Aa}\end{array}$ & $\begin{array}{l}0,114 \mathrm{Ba} \\
0,111 \mathrm{Ba}\end{array}$ & $\begin{array}{l}0,118 \mathrm{Ba} \\
0,116 \mathrm{Ba}\end{array}$ \\
\hline $\mathrm{AD}\left(\mathrm{cm}^{3} \mathrm{~cm}^{-3}\right)$ & $\begin{array}{l}05-10 \\
10-15\end{array}$ & $\begin{array}{l}0,145 \mathrm{Aa} \\
0,135 \mathrm{Ab}\end{array}$ & $\begin{array}{l}0,130 \mathrm{Ba} \\
0,131 \mathrm{Aa}\end{array}$ & $\begin{array}{l}0,121 \mathrm{Ca} \\
0,104 \mathrm{Bb}\end{array}$ \\
\hline $\mathrm{Ks}\left(\mathrm{mm} \mathrm{h}^{-1}\right)$ & $\begin{array}{l}05-10 \\
10-15\end{array}$ & $\begin{array}{l}14,70 \mathrm{Ca} \\
16,80 \mathrm{Ca}\end{array}$ & $\begin{array}{l}39,87 \mathrm{Bb} \\
55,12 \mathrm{~B} \mathrm{a}\end{array}$ & $\begin{array}{l}102,18 \mathrm{Ab} \\
207,14 \mathrm{Aa}\end{array}$ \\
\hline $\mathrm{Pt}\left(\mathrm{cm}^{3} \mathrm{~cm}^{-3}\right)$ & $\begin{array}{l}05-10 \\
10-15\end{array}$ & $\begin{array}{l}0,396 \mathrm{Ca} \\
0,392 \mathrm{Ca}\end{array}$ & $\begin{array}{l}0,417 \mathrm{~B} \mathrm{a} \\
0,424 \mathrm{~B} \mathrm{a}\end{array}$ & $\begin{array}{l}0,430 \mathrm{Ab} \\
0,448 \mathrm{Aa}\end{array}$ \\
\hline $\mathrm{Ma}\left(\mathrm{cm}^{3} \mathrm{~cm}^{-3}\right)$ & $\begin{array}{l}05-10 \\
10-15\end{array}$ & $\begin{array}{l}0,137 \mathrm{Ca} \\
0,130 \mathrm{Ca}\end{array}$ & $\begin{array}{l}0,173 \mathrm{Ba} \\
0,186 \mathrm{Ba}\end{array}$ & $\begin{array}{l}0,207 \mathrm{Ab} \\
0,239 \mathrm{Aa}\end{array}$ \\
\hline $\operatorname{Mi}\left(\mathrm{cm}^{3} \mathrm{~cm}^{-3}\right)$ & $\begin{array}{l}05-10 \\
10-15\end{array}$ & $\begin{array}{l}0,262 \mathrm{Aa} \\
0,259 \mathrm{Aa}\end{array}$ & $\begin{array}{l}0,242 \mathrm{~B} \mathrm{a} \\
0,235 \mathrm{Ba}\end{array}$ & $\begin{array}{l}0,221 \mathrm{Ca} \\
0,199 \mathrm{Cb}\end{array}$ \\
\hline
\end{tabular}

Ds: densidade do solo; RSP: resistência mecânica do solo à penetração; CA: conteúdo atual de água do solo; AD: água disponível; Ks: condutividade hidráulica saturada; Pt: porosidade total; Ma: macroporosidade; Mi: microporosidade. Médias seguidas da mesma letra, maiúscula na mesma linha e minúscula na mesma coluna, não diferem significativamente entre si pelo teste de Scott-Knott em nível de $\mathrm{p}<0,05$. 
no solo com o tratamento PD. Esses valores se encontram dentro da faixa de densidade para solos minerais, definida por Kiehl (1979) como sendo de 1,1 a $1,6 \mathrm{Mg} \mathrm{m}^{-3}$. Por outro lado, segundo esse mesmo autor, um solo com textura franco-arenosa e teor de argila em torno de $200 \mathrm{~g} \mathrm{~kg}^{-1}$, como nesse caso, para ser considerado não-compactado, deveria apresentar valores de Ds abaixo de $1,40 \mathrm{Mg} \mathrm{m}^{-3}$. Entretanto, não há consenso entre os pesquisadores quanto aos valores críticos de densidade do solo. Torres \& Saraiva (1999) afirmaram que a densidade varia de $1,0 \mathrm{Mg} \mathrm{m}^{-3} \mathrm{em}$ solos argilosos sob mata a $1,45 \mathrm{Mg} \mathrm{m}^{-3}$ quando muito compactado; da mesma forma, varia de 1,25 a $1,70 \mathrm{Mg} \mathrm{m}^{-3}$, respectivamente, para solos arenosos sob mata e quando compactados. Já Camargo \& Alleoni (1997) afirmam ser crítica, para solos argilosos e franco-argilosos, a densidade de 1,55 $\mathrm{Mg} \mathrm{m}^{-3}$. Neste experimento, verificaram-se densidades do solo mais elevadas em solo sob plantio direto (PD), seguido pelo solo sob pastagem (PC), tendo o Cerrado nativo pastejado $(\mathrm{CP})$ os menores valores para este atributo.

Verifica-se ainda a ocorrência de maiores valores de densidade do solo na camada superior amostrada, no caso do solo sob CP, pois a diferença de $0,05 \mathrm{Mg} \mathrm{m}^{-3}$ entre as Ds das camadas de 5-10 e 10-15 cm neste tratamento foi significativa a 0,05. Isso, provavelmente, tenha ocorrido devido à livre visitação do gado, acarretando o pisoteio do solo; aliado a isso, há a baixa variabilidade (CV) observada para este atributo, que pode influenciar fortemente os resultados do teste de médias, uma vez que, neste caso, pequena variação ocorrida nos valores da Ds pode resultar em diferenças significativas.

Por outro lado, não houve diferença significativa dos valores médios de Ds das camadas de solo estudadas em área sob pastagem, o que reforça a hipótese de que o uso do solo por longo período de tempo propicia homogeneização das camadas em relação aos valores desse atributo.

Com relação à RSP, seus valores médios observados nas camadas estudadas (Quadro 2) e a distribuição dos valores médios ao longo do perfil de 0-300 mm (Figura 1) confirmaram as tendências observadas para a densidade do solo, ou seja, com valores mais elevados na camada de $5-10 \mathrm{~cm}$.

A RSP tem sido mais sensível em captar a variabilidade vertical do efeito do uso sobre o solo, uma vez que as médias observadas, nas duas profundidades, independentemente do uso, foram consideradas diferentes estatisticamente. Os maiores valores de RSP na camada de $10-15 \mathrm{~cm}$, observados em PD, caracterizam o efeito do uso de máquinas, mesmo que em pequena intensidade; já os maiores valores de RSP na camada de 5-10 cm nos tratamentos PC e CP evidenciaram o efeito do pisoteio do gado, que se faz mais intenso nas primeiras camadas do solo.

Considerando o valor crítico de 2,0 MPa proposto por Taylor et al. (1966) e amplamente aceito no meio

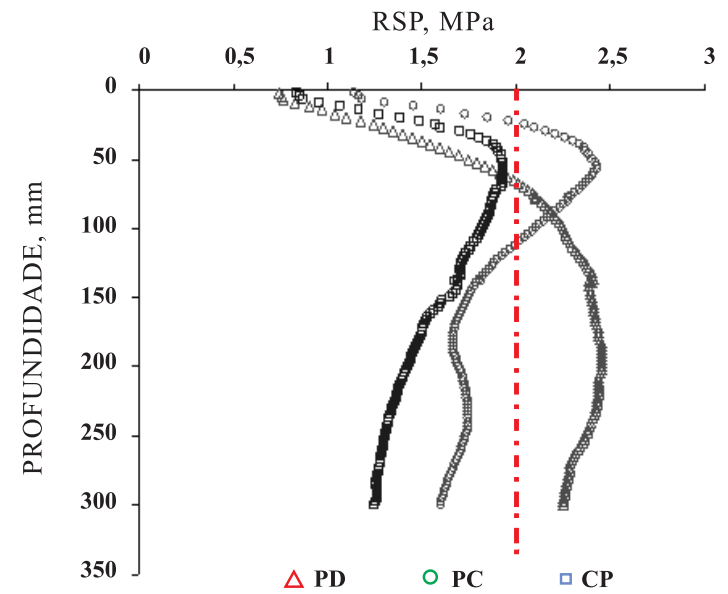

Figura 1. Curvas de resistência mecânica do solo à penetração média (RSP), em função da profundidade, observadas em Latossolo Vermelho-Amarelo distrófico sob plantio direto (PD), pastagem cultivada (PC) e Cerrado nativo pastejado (CP).

científico, verifica-se que nos solos sob PD e na camada superior do solo sob PC, provavelmente, ocorreriam restrições ao crescimento radicular, embora sejam as gramíneas menos suscetíveis aos efeitos da compactação (Silva et al., 2006). Como ocorre com a Ds, também não há consenso quanto ao valor crítico de RSP; Richart et al. (2005) sugerem, em sua revisão, valores variando de 1,0 a 5,0 MPa como limites críticos para RSP em diferentes tipos de solo.

Nota-se também aparente contradição no tocante aos valores médios de RSP observados para o solo sob $\mathrm{PD}$, considerando-se que os valores de densidades nas duas camadas foram estritamente iguais, enquanto a RSP foi, em média, 0,32 MPa menor na camada superior (Quadro 2).

Observando a figura 1, percebe-se que a RSP em $\mathrm{PD}$ não só se apresenta menor na camada de $5-10 \mathrm{~cm}$, como seus valores na profundidade de $0-5 \mathrm{~cm}$ foram menores até mesmo que aqueles verificados na mesma camada em CP. Isso deve-se ao fato de que o revolvimento da camada superficial, pela passagem de grade na semeadura do milheto e pelos discos de corte da semeadora de milho sobre os resíduos da soja, provavelmente, tenha rompido ligações macroestruturais do solo, que potencializariam incremento na RSP; todavia, o tempo transcorrido foi suficiente para acomodação do solo no sentido de atingir as densidades observadas, mas não para reconstruir a sua estrutura.

Por outro lado, o conteúdo de água do solo no momento da amostragem CA (Quadro 2) foi maior no $\mathrm{PD}$, o que pode ter minimizado o efeito da RSP, que, então, apresentaria valores subestimados nessa condição, uma vez que existe correlação inversa entre RSP e CA (Mckyes, 1985; Lima et al., 2007). 
A água disponível $(\mathrm{AD})$ foi maior no solo sob $\mathrm{PD}$, na camada de $5-10 \mathrm{~cm}$, em relação aos tratamentos $\mathrm{PC}$ e CP, estando de acordo com os resultados encontrados por Marchão et al. (2007) em um Latossolo Vermelho de textura argilosa. Entretanto, enquanto neste experimento houve incremento de $\mathrm{AD}$ com a elevação da Ds, no estudo realizado pelos autores citados anteriormente não foi observado esse comportamento. Isso se deve ao fato de que a elevação na densidade em solos de textura mais leve, como no presente caso, possivelmente, produza incremento proporcionalmente maior na capacidade de retenção de água desses solos, como também relataram Cavichiolo et al. (2005), do que naqueles de textura argilosa, devido ao incremento no volume de microporos, que possibilita maior retenção de água por capilaridade, reduzindo a percolação e evaporação.

Os solos sob PD e PC tiveram decréscimos de 3,0 a $12,5 \%$ na porosidade total $(\mathrm{Pt})$ e de 16,4 a $45,6 \%$ na macroporosidade (Ma), em relação aos valores observados para os solos sob CP, indicando alteração estrutural do solo pela intervenção antrópica, evento observado por diversos autores que relatam a suscetibilidade da porosidade, em especial da macroporosidade, aos efeitos do manejo, de Derpsch et al. (1986) a Silva et al. (2006). Os valores de porosidade de aeração (Ma) observados em solo sob PD são similares aos geralmente adotados como restritivos para o crescimento e a produtividade da maioria das culturas, que, conforme Tormena et al. (2002), situam-se entre 10 e $15 \%$.

No solo sob CP, observa-se aumento da Pt e da Ma em profundidade, fato que geralmente não ocorre em solos sob condições naturais, conforme resultados encontrados por Spera et al. (2004), entre outros. Nesse caso, esse comportamento é explicado pela compactação da camada de $5-10 \mathrm{~cm}$, provocada pelo pisoteio do gado que pasteja na área de Cerrado nativo.

A microporosidade (Mi) teve acréscimo de 18,5 e $30,1 \%$ nas camadas de $5-10$ e $10-15 \mathrm{~cm}$, respectivamente, no solo em $\mathrm{PD}$, e de 9,5 e $18,1 \%$ para o solo sob PC, ambos em relação aos valores observados em CP. Como discutido para os atributos Ma e PT, também se percebe o efeito do pisoteio do gado, criando gradiente vertical de $\mathrm{Mi}$, contrariamente ao esperado em solos sob Cerrado natural. Os resultados observados neste experimento contrariam, em parte, os de Spera et al. (2004), que não encontraram efeito do pisoteio do gado sobre valores da Mi em sistemas integrados lavoura-pecuária.

Em relação à permeabilidade do solo, a análise dos valores médios de Ks (Quadro 2) reforça a idéia de alteração estrutural dos solos pelo uso, principalmente em relação a forma, tamanho e continuidade de poros, que afetariam a dinâmica da água no solo (Souza \& Alves, 2003). A condutividade hidráulica do solo saturado (Ks) foi drasticamente afetada pelo manejo, tendo ocorrido reduções de 60,1 a $91,9 \%$ nos seus valores nos tratamentos $\mathrm{PC}$ e $\mathrm{PD}$, respectivamente, comparando-se à Ks observada em CP. Isso se deve à redução da Ma, em razão do aumento da compactação do solo. Essa redução na condutividade hidráulica do solo, sob efeito da compactação, é relatada por Oliveira et al. (1998). A Ks teve comportamento inverso ao da RSP, como era de se esperar, pois, enquanto a primeira diminui, em razão da menor quantidade de poros para infiltração de água, a segunda tende a aumentar.

Neste experimento verificaram-se correlações significativas para experimentos realizados em campo. As menores correlações ocorreram entre Ds e CA $(0,65), \operatorname{RSP}$ e Ks $(-0,66)$ e RSP e Ds $(0,69)$, ao passo que as maiores correlações foram observadas entre Ds e Ks $(-0,82)$, DS e Ma $(-0,85)$ e entre Ma e Ks $(0,86)$. Estes coeficientes de correlação se assemelham aos obtidos por Silva et al. (2006) para Latossolo VermelhoAmarelo em experimento de laboratório, exceto em relação à RSP e Ds, que, evidentemente, em condições laboratoriais foram consideravelmente maiores.

Souza \& Alves (2003), estudando um Latossolo Vermelho distrófico típico, argiloso, sob plantio direto, cultivo mínimo, pastagem de Brachiaria brizantha e Cerrado nativo, relataram reduções na condutividade que variaram de 54 a $82 \%$ em relação ao Cerrado nativo. Contudo, além das diferenças de amplitude dos efeitos observados sobre a condutividade hidráulica saturada, nos dois trabalhos, causadas, provavelmente, pela diferença de textura entre os solos estudados, esses autores observaram redução de $\mathrm{Ks}$ em profundidade no Cerrado e em $\mathrm{PD}$ e, além de encontrarem maior redução de Ks no solo sob pastagem, não verificaram um gradiente de condutividade hidráulica. No presente estudo, entretanto, a Ks teve acréscimo em profundidade no solo sob CP e sob PC, confirmando a existência de uma camada adensada de $5-10 \mathrm{~cm}$. Ressalta-se que o solo sob pastagem se apresentou com condutividade hidráulica saturada superior à mostrada pelo solo sob $\mathrm{PD}$, nas duas camadas estudadas.

A inversão dos gradientes de permeabilidade dos solos, ou seja, condutividade decrescente em profundidade (Souza \& Alves, 2003) e crescente, como se observa nos solos sob CP e PC deste trabalho, justifica-se em razão da localização da camada com menor porosidade de drenagem. Nos solos com elevado teor de areia, a energia de compactação aplicada se concentra-se nas camadas superficiais, enquanto nos solos com maior teor de argila ela é distribuída em profundidade, uma vez que a compressividade do solo depende do teor e tipo de argila que determina o alcance e a persistência da compactação (Horn, 1988; Seybold et al., 1999).

O modelo de Van Genuchten se apresentou adequado para explicar a retenção de água no solo (Figura 2). O deslocamento ascendente das curvas de $\mathrm{PC}$ e PD, em relação às curvas de $\mathrm{CP}$, observado nesta figura, deve-se às alterações na porosidade, promovidas pelo uso e manejo do solo, que acarretaram sua compactação, reduzindo a porosidade drenável e 


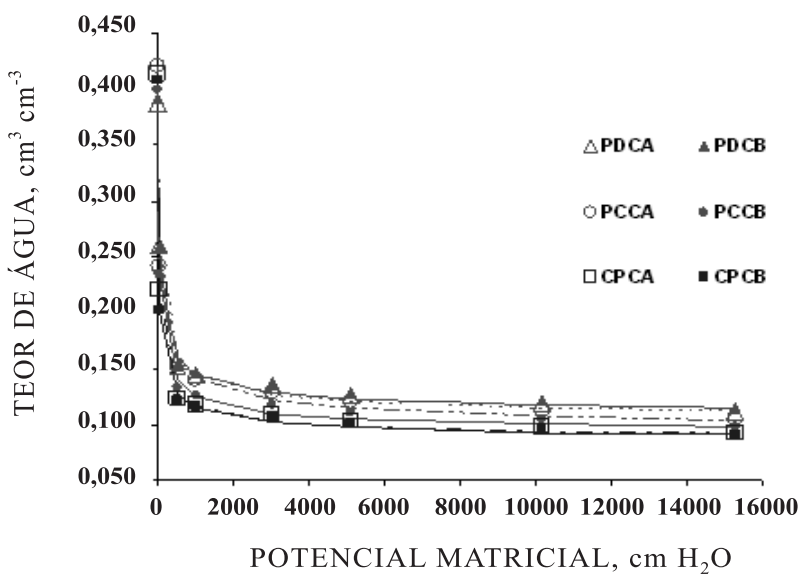

Figura 2. Curvas de retenção de água de um Latossolo Vermelho-Amarelo distrófico sob plantio direto (PD), pastagem cultivada (PC) e Cerrado nativo pastejado (CP), nas profundidades de 5-10 cm (CA) e 10-15 cm (CB).

incrementando a porosidade de retenção de água, como relatam Bertol et al. (2001). Assim, corroborado por Resende et al. (1999), subentende-se que, em solos de textura mais leve, um pequeno acréscimo na densidade seria benéfico, por promover maior capacidade de reter e de disponibilizar água. Isso se evidencia no presente estudo, tendo em vista que os solos sob PC e PD apresentaram menores valores de Ma e maiores de Mi e curvas de retenção de água no solo, com tendência de deslocamento ascendente, ou seja, considerando-se a mesma tensão aplicada, o teor de água retido no solo nesses dois tratamentos foi superior ao do tratamento CP.

\section{CONCLUSÕES}

1. As maiores alterações nos atributos físico-hídricos do solo ocorreram na área sob plantio direto, que, ainda assim, apresentaram níveis considerados favoráveis ao desenvolvimento de plantas.

2. O pisoteio de animais, tanto em área sob pastagem cultivada como em área de Cerrado nativo pastejado, promoveu alterações mais severas nos atributos físico-hídricos da camada de 5 a $10 \mathrm{~cm}$.

3. A resistência do solo à penetração, a macroporosidade e a condutividade hidráulica saturada se apresentaram mais sensíveis e são, portanto, melhores indicadores dos efeitos do uso e manejo do solo.

4. O plantio direto e o uso com pastagem cultivada promoveram alterações nas condições físico-hídricas do LVAd de textura franco-arenosa, elevando a sua capacidade de armazenamento de água.

\section{LITERATURA CITADA}

AZEVEDO, E.C. Uso da geoestatística e de recursos de geoprocessamento no diagnóstico da degradação de um solo argiloso sob pastagem no Estado de Mato Grosso. Campinas, Universidade Estadual de Campinas, 2004. 158p. (Tese de Doutorado)

BERTOL, I.; BEUTLER, J.F.; LEITE, D. \& BATISTELA, O. Propriedades físicas de um Cambissolo Húmico afetado pelo tipo de manejo do solo. Sci. Agríc., 58:555-560, 2001.

BIANCHINI, A.; MAIA, J.C.M.; MAGALHAES, P.S.G.; CAPELLI, N. \& UMEZU, C.K. Penetrógrafo eletrônico automático. R. Bras. Eng. Agric. Amb., 6:332-336, 2002.

CAMARGO, O.A. \& ALLEONI, L.R.F. Compactação do solo e o desenvolvimento das plantas. Piracicaba, 1997. 132p.

CAVICHIOLO, S.R.; DEDECEK, R.A. \& GAVA, J. L. Modificações nos atributos físicos de solos submetidos a dois sistemas de preparo em rebrota de Eucalyptus saligna. R. Árvore, 29:571-577, 2005.

CENTURION, J.F.; CARDOSO, J.P. \& NATALE, W. Efeito de formas de manejo em algumas propriedades físicas e químicas de um Latossolo Vermelho em diferentes agroecossistemas. R. Bras. Eng. Agric. Amb., 5:254-258, 2001.

D’ANDRÉA, A.F.; SILVA, M.L.N.; CURI, N. \& FERREIRA, M.M. Atributos de agregação indicadores da qualidade do solo em sistemas de manejo na região dos Cerrados no sul do Estado de Goiás. R. Bras. Ci. Solo, 26:1047-1054, 2002.

DA ROS, C.O.; SECCO, D.; FIORIN, J.E.; PETRERE, C.; CADORE, M.A. \& PASA, L. Manejo do solo a partir de campo nativo: Efeito sobre a forma de estabilidade da estrutura ao final de cinco anos. R. Bras. Ci. Solo, 21:241247, 1997.

DERPSCH, R.; SIDIRAS, N. \& ROTH, C.H. Results of studies made from 1977 to 1984 to control erosion by cover crops and no-tillage techniques in Paraná, Brazil. Soil Till. Res., 8:253-263, 1986.

DOURADO-NETO, D.; NIELSEN, D.R.; HOPMANS, J.W.; REICHARDT, K.; BACCHI, O.O.S. \& LOPES, P.P. Software to model soil water retention curves (SWRC, version 2.00). Sci. Agric., 57:191-192, 2000.

EMPRESA BRASILEIRA DE PESQUISA AGROPECUÁRIA EMBRAPA. Centro Nacional de Pesquisa de Solos. Manual de métodos de análise de solo. 2. ed. Rio de Janeiro, 1997. 212p.

FERREIRA, D.F. Análise estatística por meio do SISVAR (Sistema para Análise de Variância) para Windows versão 4.0. In: REUNIÃO ANUAL DA REGIÃO BRASILEIRA DA SOCIEDADE INTERNACIONAL DE BIOMETRIA, 45., São Carlos, 2000. Anais. São Carlos, Universidade Federal de São Carlos, 2000. p.255-258.

FREITAS-JÚNIOR, E. \& LUCHIARI-JÚNIOR, A. Manejo: aspectos físicos. In: PEREIRA, V.P.; FERREIRA, M.E. \& CRUZ, M.C.P., eds. Solos altamente suscetíveis à erosão. Jaboticabal, FCAV, 1994. p.113-122. 
GRADWELL, M.W. Soil moisture deficiencies in puddled pastures. N. Z. J. Agric. Res., 9:127-136, 1966.

HORN, R. Compressibility of arable land. Catena, 11:53-71, 1988.

KIEHL, E.J. Manual de edafologia. São Paulo, Ceres, 1979. 262p.

KÖPPEN, W. Climatologia: con un estudio de los climas de la tierra. México, Fondo de Cultura Econômica, 1948. 479p.

LIMA, C.A.G. \& SILANS, A.P. Variabilidade espacial da infiltração de água no solo. Pesq. Agropec. Bras., 34:2311 2320,1999 .

LIMA, C.L.R.; REICHERTI, J.M.; REINERTI, D.J.; SUZUK, L.E.A.S. \& DALBIANCO, L. Densidade crítica ao crescimento de plantas considerando água disponível e resistência à penetração de um Argissolo Vermelho distrófico arênico. Ci. Rural, 37:1166-1169, 2007.

MARCHÃO, R.L.; BALBINO, L.C.; SILVA, E.M.; SANTOS JUNIOR, J.D.G.; SÁ, M.A.C.; VILELA, L. \& BECQUER, T. Qualidade física de um Latossolo Vermelho sob sistemas de integração lavoura-pecuária no Cerrado. Pesq. Agropec. Bras., 42:873-882, 2007.

McKYES, E. Soil loosening and manipulation. In: Cutting and tillage developments in agricultural engineering. 7., New York, Elsevier, 1985. p. 87-104.

MELO, V.F. \& SILVA, J.R.C. Propriedades físicas de um Latossolo Amarelo álico, em áreas sob cultivo e vegetação natural de Cerrado. In: CONGRESSO BRASILEIRO DE CIÊNCIA DO SOLO, 25., Viçosa, 1995. Anais. Viçosa, MG, Sociedade Brasileira de Ciência do Solo, 1995. p.1890-1892.

MORAES, A. \& LUSTOSA, S.B.C. Efeito do animal sobre as características do solo e a produção da pastagem. In: SIMPÓSIO SOBRE AVALIAÇÃO DE PASTAGENS COM ANIMAIS, Maringá 1997. Anais. Maringá, Universidade Estadual de Maringá, 1997. p.129-149.

NIELSEN, D.R.; BIGGAR, J.W. \& ERH, K.T. Spatial variability of field-measured soil water properties. Hilgardia, 42:251260, 1973

OLIVEIRA, C.V.; BAHIA, V.G. \& PAULA, M.B. Compactação do solo devido à mecanização florestal: Causas, efeitos e práticas de controle. Inf. Agropec., 191:46-48, 1998.

OLIVEIRA, G.C. Alterações estruturais e comportamento compressivo de um Latossolo submetido a diferentes sistemas de manejo por 20 anos no Cerrado. Lavras, Universidade Federal de Lavras, 2002. 78p. (Tese de Doutorado)

RESENDE, M.; CURI, N.; REZENDE, S.B. \& CORRÊA, G.F. Pedologia: Base para distinção de ambientes. 3.ed. Viçosa, MG, NEPUT, 1999. 304p.
RICHART, A.; TAVARES FILHO, J.; BRITO, O.R.; LLANILLO, R.F. \& FERREIRA, R. Compactação do solo: Causas e efeitos. Semina: Ci. Agric., 26:321-344, 2005.

SEYBOLD, C.A.; HERRICK, J.E. \& BREJDA, J.J. Soil resilience: A fundamental component of soil quality. Soil Sci., 164:224-234, 1999.

SILVA, G.J.; MAIA, J.C.S. \& BIANCHINI, A. Crescimento da parte aérea de plantas cultivadas em vaso, submetidas à irrigação subsuperficial e a diferentes graus de compactação de um Latossolo Vermelho-Escuro distrófico. R. Bras. Ci. Solo, 30:31-40, 2006.

SILVA, J.E. \& RESCK, D.V.S. Respostas fisiológicas da soja ao déficit hídrico em dois solos de Cerrado. Pesq. Agropec. Bras., 16:669-675, 1981.

SILVA, S.R.; BARROS, N.F. \& COSTA, L.M. Atributos físicos de dois Latossolos afetados pela compactação do solo. R. Bras. Eng. Agric. Amb., 10:842-847, 2006.

SOARES, J.L.N.; ESPINDOLA, C.R. \& CASTRO, S.S. Alteração física e morfológica em solos cultivados sob sistema tradicional de manejo. R. Bras. Ci. Solo, 29:1005-1014, 2005 .

SOUZA, Z.M. \& ALVES, M.C. Movimento de água e resistência à penetração em um Latossolo Vermelho distrófico de Cerrado, sob diferentes usos e manejos. R. Bras. Eng. Agríc. Amb., 7:18-23, 2003.

SPERA, S.T.; SANTOS, H.P.; FONTANELI, R.S. \& TOMM, G.O. Efeitos de sistemas de produção de grãos envolvendo pastagens sob plantio direto nos atributos físicos de solo e na produtividade. R. Bras. Ci. Solo, 28:533-542, 2004.

TAYLOR, H.M.; ROBERTSON, G.M. \& PARKER, J.J. Soil strength root penetration relations for medium to coarse textured soil materials. Soil Sci., 102:18-22, 1966.

TORMENA, C.A.; BARBOSA, M.C.; COSTA, A.C.S. \& GONÇALVES, A.C.A. Densidade, porosidade e resistência à penetração em Latossolo cultivado sob diferentes sistemas de preparo do solo. Sci. Agric., 59:795-801, 2002.

TORRES, E. \& SARAIVA, O.F. Camadas de impedimento mecânico do solo em sistemas agrícolas com soja. Londrina, Embrapa-CNP Soja, 1999. 58p. (Circular Técnica, 23)

van GENUCHTEN, M.T. A closed form equation for predicting hydraulic conductivity of unsaturated soils. Soil Sci. Soc. Am. J., 44:892-898, 1980.

VIEIRA, M.J. Propriedades físicas do solo. In: FUNDAÇÃO INSTITUTO AGRONÔMICO DO PARANÁ. Plantio direto no Estado do Paraná. Londrina, 1981. p.19-30. (Circular, 23) 